\title{
RATE SENSITIVITY OF PLASTIC FLOW AND IMPLICATIONS FOR YIELD-SURFACE VERTICES
}

\author{
JWo PaN \\ Stress Analysis and Fracture Section, Battelle Columbus Laboraturies, Culumbus. OH 43201, U.S.A. \\ and \\ JAMES R. RICE \\ Division of Applied Sciences, Harvard University, Cambridge, MA 02138, U.S.A.
}

(Receiced I April 1982; in revised form 29 Nocember 1982)

\begin{abstract}
Ahatract-When crystalline slip is considered as the micromechanism of plastic deformation in polycrystals, and the slip process is assumed to be rate-independent, the formation of a vertex is predicted on the current yield surface in stress-space. Experiments which were conducted to confirm the existence of such a vertex are known to have produced ambiguous results; e.g. a superimposed shear during compressive loading was found to produce an initially elastic response. If we consider that the crystalline slip process is slightly rate-dependent, however, then we can furnish a reasonable explanation for this elastic behavior in shear. We illustrate the point by considering first a single crystal model undergoing double slip, and then a polycrystal model based on the slip concepts of Batdorf and Budiansky. The results presented, based on the assumption that the slip process is not rate-independent, but rather at least slightly ratedependent, give qualitative agreement, and reasonable quantitative agreement, to experimental results for superposed shear during compressive loading. They also suggest that the actual rate sensitivity of plastic flow may be central to understanding the ambiguous conclusions from experimental attempts to find yield surface vertices.
\end{abstract}

\section{INTRODUCTION}

The principal micromechanism of plastic flow in metals at low temperatures is crystalline slip via dislocation motion. Furthermore, it seems normally reasonable to adopt a Schmid-like description such that the slip rate on a given crystalline slip system, or the average dislocation velocity on that system, depends on the current existing stress state only through a dependence on the resolved shear stress on that system. In the rate-independent idealization of plastic flow, this Schmid description reduces to the local "yield criterion" that a given slip system within a given element of crystal yields when a critical resolved shear stress is attained. Now, as Hill[1] has remarked, theories of macroscopic rate-independent plasticity of polycrystals, based on the concept of Schmid-like crystalline slip in each grain, lead inevitably to the prediction of a pointed vertex on the current yield surface in (macroscopic) stress space. This contrasts with the classical rate-independent plasticity formulations (such as $J_{2}$ flow theory or, equivalently, the Prandtl-Reuss equations and their generalizations) which postulate a smooth yield surface at the current loading point. The reason for the generality of the predicted vertex structure, when flow occurs by the slip micromechanism, is easy to understand. An explanation is given in Appendix 1. Essentially, the macroscopic yield surface can be regarded as the inner envelope of an unbounded number of planar yield surfaces, representing critical shear conditions for each slip system at each (locally crystalline) element of material; see Fig. 1. At conditions well into the plastic range, the collection of planes passing through the current stress state (i.e. corresponding to plastically active locations and slip systems) encompasses a wide range of orientations, and hence a vertex develops as indicated.

Experiments, however, have not led to an unambiguous resolution of the question as to whether vertices exist. Some workers have interpreted their results as being supportive of vertex formation, others as being not so; see the reviews by Paul[2] and, more recently, Hecker [3]. The experiments divide into two types. In one, a vertex is sought directly on the yield surface. In the other, one looks at how the direction of the plastic strain increment relates to that of the stress increment for a zigzag loading path, for example, in a combined torsion-compression test.

We show that a very small plastic strain-rate sensitivity, of a magnitude which could go essentially unnoticed in direct tension or shear testing over a modest range of rates, is sufficient 


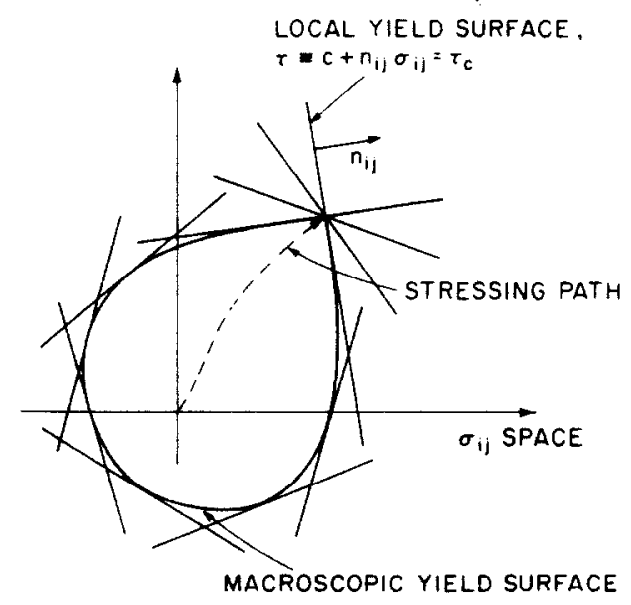

Fig. 1. The macroscopic yield surface in stress space is the inner envelope of all (planar) yield surfaces corresponding to critical shear stress conditions on individual crystalline slip systems at local poinis of a polycrystalline array. All local surfaces corresponding to points and systems which are plastically active must contain the current stress state, and hence a pointed vertex structure is developed.

to alter significantly some consequences of the vertex formation predicted by the rateindependent models. We note that it is now generally accepted that, as expressed in dislocation dynamics studies of Johnston and Gilman $[4,5]$ and others, plastic deformation in metals is an inherently rate-dependent process. Rice $[6,7]$ has established a rigorous mathematical framework, analogous to that of Hill[1] for the rate-independent case, for the incorporation of the dislocation dynamics viewpoint of slip in constitutive modelling for general stress states and loading paths. He shows further that if the Schmid-like assumption is made that the shearing rate on a given slip system depends on the local stress state only through the shear stress resolved onto that system, then at each instant in a history of deformation, the current (macroscopic) plastic strain rate depends only on the current stress, and is derivable from a scalar "flow potential" by differentiation with respect to the corresponding stress component. As remarked by Rice [6], the macroscopic plastic strain rate is a continuous function of stress if the local slip system shearing rates vary continuously with resolved shear stresses and, consequently, a sudden change of a stress rate $\dot{\boldsymbol{\sigma}}$ will not alter the plastic strain rate $\dot{\boldsymbol{\epsilon}}^{\mathrm{P}}$ abruptly. This suggests that rate-dependence, even if slight, cannot lead to a sharp vertex structure.

A number of experiments in the form of torsion-compression tests on thin-walled tubes have been carried out. Those by Budiansky et al.[8] have received particular attention in the literature. Those investigators found that, during compressive loading, an imposed shear produced an initially elastic response. The elastic response suggests that the current yield surface, in the framework of rate-independent plasticity, may be smooth. The lack of an evident vertex effect has been interpreted by some as a failure of the slip-based concepts. However, by considering the material's plastic strain rate sensitivity, we offer an alternative explanation which suggests that the slip-based concepts may themselves be sound and do, when implemented in a rate-dependent framework, lead to the conclusion that initial shear response, superposed on tension or compression loading, is elastic.

\section{A DOUBLE SLIP MODEL FOR SINGLE CRYSTALS}

It is commonly observed that a double slip deformation pattern develops under simple tension loading of a single crystal. To illustrate simply vertex formation on the yield surface within a rate-independent approach, and to demonstrate later the effects of rate sensitivity, we consider a single crystal which has been deforming in a (symmetrical) double slip pattern under simple tension stress $\sigma_{11}$ and imagine that a small shearing stress $\sigma_{12}$ is superposed. Asaro [9] used this double slip model to study the stability of rate-independent plastic flow in crystals. He comments on the "geometrical softening" $[10,11]$ that can arise in single crystals, e.g. from rotation of the lattice due to grip constraint in tensile loading. The schematic picture of 
the double slip system is shown in Fig. 2(a). This figure shows the idealized two-dimensional double-slip model which has the slip vectors $s_{1}$ and $s_{2}$, and the slip plane normals, $n_{1}$ and $n_{2}$, on the plane of the drawing. However, when the real crystal undergoes double slip with the tensile axis along the $x_{1}$ direction, both the slip vectors $s_{1}$ and $s_{2}$ are in the plane of the drawing, but the slip plane normals, $\mathbf{n}_{1}$ and $\mathbf{n}_{2}$, are tilted outward.

We consider the two-dimensional model and idealize the problem such that rotations of lattice directions are neglected. A more precise analysis that includes the rotations is given in Appendix 2. It leads to conclusions which are similar to those following, and reduces to the following when the crystal hardening modulus exceeds greatly the stress level. Thus the resolved shear stresses $\tau_{1}$ and $\tau_{2}$ on the two systems and their rates are given by

$$
\begin{aligned}
& \tau_{i}=\mathbf{n}_{i} \cdot \boldsymbol{\sigma} \cdot \mathbf{s}_{i}, i=1,2 \\
& \dot{\tau}_{i}=\mathbf{n}_{i} \cdot \dot{\boldsymbol{\sigma}} \cdot \mathbf{s}_{i}, i=1,2
\end{aligned}
$$

where $\sigma$ is the true or Cauchy stress, and $\dot{\sigma}$ is its time rate.

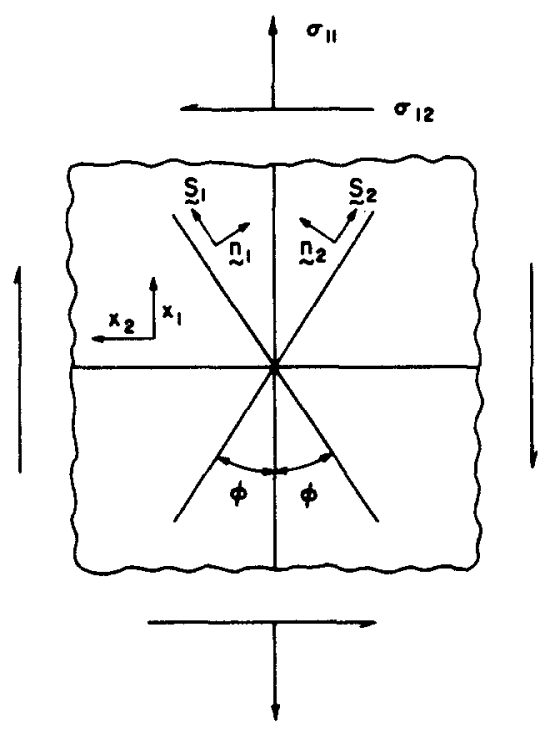

(a)

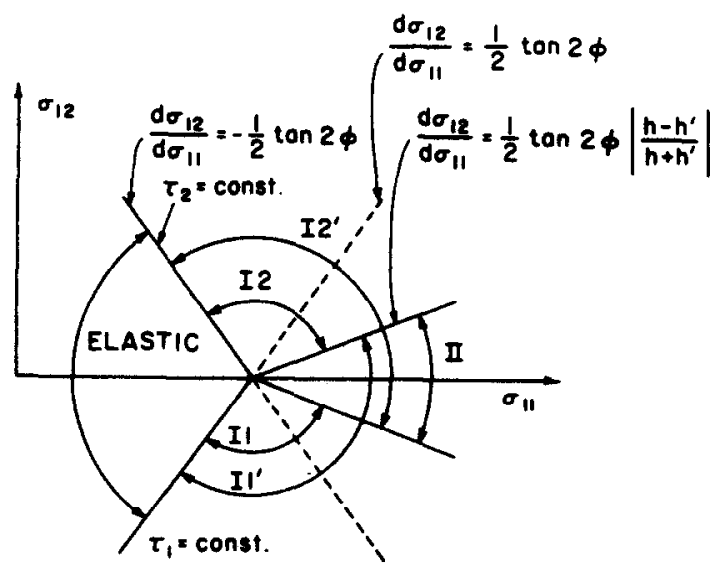

(b)

Fig. 2(a). The idealized two-dimensional model of a single crystal undergoing a double slip pattern. (b). The crystal response in stress space at the inception of shear is shown. The lines marked $\tau_{2} \approx$ const. and $\tau_{1}=$ const. define the yield surface. 
In terms of the angle $2 \phi$ between the slip planes as depicted in Fig. 2(a), eqn (2) can be expressed as:

$$
\begin{aligned}
& \dot{\tau}_{1}=\frac{1}{2} \dot{\sigma}_{11} \sin 2 \phi-\dot{\sigma}_{12} \cos 2 \phi \\
& \dot{\tau}_{2}=\frac{1}{2} \dot{\sigma}_{11} \sin 2 \phi+\dot{\sigma}_{12} \cos 2 \phi .
\end{aligned}
$$

Since the deformation is assumed to be homogeneous, the plastic strain rate $\dot{\boldsymbol{\epsilon}}^{p}$ is related to crystalline slip rates $\dot{\gamma}_{\mathrm{i}}$ by

$$
\dot{\boldsymbol{\epsilon}}^{p}=\sum_{i=1}^{2} \frac{1}{2}\left(\mathbf{n}_{i} \mathbf{s}_{i}+\mathbf{s}_{i} \mathbf{n}_{i}\right) \dot{\gamma}_{i}
$$

in component form this becomes

$$
\begin{gathered}
\dot{\epsilon}_{11}^{p}=\frac{1}{2} \sin 2 \phi\left(\dot{\gamma}_{1}+\dot{\gamma}_{2}\right) \\
\dot{\epsilon}_{12}^{p}=\frac{1}{2} \cos 2 \phi\left(\dot{\gamma}_{2}-\dot{\gamma}_{1}\right) \\
\dot{\epsilon}_{22}^{p}=-\dot{\epsilon}_{11}^{p} .
\end{gathered}
$$

The rate-independent constitutive law governing multi-slip processes in single crystals is given by (e.g. Hill[12])

$$
i_{i} \leq \sum_{j=1}^{2} h_{i j} \dot{\gamma}_{j,} i=1,2
$$

where the $h_{i 1}$ are hardening moduli; $h_{11}$ and $h_{22}$ are self-hardening moduli, and $h_{12}$ and $h_{21}$ are cross-hardening (or latent hardening) moduli. Equality holds when the $i$ th slip system is active and inequality otherwise. Consistently with the presumed symmetrical double slip, we assume

$$
\begin{aligned}
& h_{11}=h_{22} \equiv h>0 \\
& h_{12}=h_{21} \equiv h^{\prime} \geq 0 .
\end{aligned}
$$

At the instant of tensile loading $\sigma_{11}$ that is being considered, before shear application, both systems have identical strengths and hence the yield surface has the vertex structure (formed by surfaces $\tau_{1}=$ constant and $\tau_{2}=$ constant) illustrated in Fig. 2(b). We assumed that $\phi<45^{\circ}$.

We now investigate loading regimes in the $\sigma_{11}, \sigma_{12}$ plane corresponding to continued double slip and to single slip, respectively, presuming that the considered stress increments $\dot{\sigma}_{11}, \dot{\sigma}_{12}$ form a vector pointing outward from the yield surface (otherwise, they cause only elastic response). Suppose, for example, that after the inception of shear loading $\sigma_{12}$, slip system 2 is active and slip system 1 is inactive; of course, both slip systems were activated by the tensile stressing $\sigma_{11}$ before $\sigma_{12}$ was applied. From $\dot{\tau}_{1} \leq h_{12} \dot{\gamma}_{2}=h_{12} \dot{\tau}_{2} / h_{22}=h^{\prime} \dot{\tau}_{2} / h$, which follows from (4), there results the inequality

$$
\dot{\sigma}_{12} \geq \frac{1}{2} \tan 2 \phi \frac{h-h^{\prime}}{h+h^{\prime}} \dot{\sigma}_{i 1}
$$

when eqns ( $2 a)$ and $(2 b)$ are used.

Similarly, for the case that slip system 1 is active and slip system 2 is inactive, one must 
have $\dot{\tau}_{2} \leq h^{\prime} \dot{\tau}_{1} / h$, or

$$
\dot{\sigma}_{12} \leq-\frac{1}{2} \tan 2 \phi \frac{h-h^{\prime}}{h+h^{\prime}} \dot{\sigma}_{11}
$$

On the other hand, the condition for both 1 and 2 to be active, obtained by writing (4) as an equality, solving for $\dot{\gamma}_{1}, \dot{\gamma}_{2}$, and requiring that $\dot{\gamma}_{1}>0, \dot{\gamma}_{2}>0$ is that simulatneously ( $h \dot{\tau}_{1}-$ $\left.h^{\prime} \dot{\tau}_{2}\right) /\left(h^{2}-h^{\prime 2}\right) \geq 0,\left(h \dot{\tau}_{2}-h^{\prime} \dot{\tau}_{1}\right) /\left(h^{2}-h^{\prime 2}\right) \geq 0$. From eqns $(2 \mathrm{a})$ and $(2 \mathrm{~b})$ these are equivalent to the continued inequality

$$
-\frac{1}{2} \tan 2 \phi \dot{\sigma}_{11} \leq \frac{h+h^{\prime}}{h-h^{\prime}} \dot{\sigma}_{12} \leq \frac{1}{2} \tan 2 \phi \dot{\sigma}_{11}
$$

which can be met only if $\dot{\sigma}_{11} \geq 0$ and

$$
\left|\dot{\sigma}_{12}\right| \leq \frac{1}{2} \tan 2 \phi \frac{\left|h-h^{\prime}\right|}{h+h^{\prime}} \dot{\sigma}_{11}
$$

The domains of the $\sigma_{11}, \sigma_{12}$ plane delineated by inequalities (5), (7b) and (6) are distinct bordering regions, if $h>h^{\prime}$. But the regions overlap when $h<h^{\prime}$. This overlap means that when $h<h^{\prime}$, a specification of the stress rates $\dot{\sigma}_{11}, \dot{\sigma}_{12}$ does not determine uniquely whether single or double slips result; the character of the slip is, however, determined uniquely by specification of $\dot{\sigma}_{11}, \dot{\sigma}_{12}$ when $h>h^{\prime}$ since there is then no overlap.

The nature of the response at the inception of shear loading is illustrated in Fig. 2(b). The two lines marked $\tau_{2}=$ const., $\tau_{1}=$ const. define the yield locus and the domain of elastic response is indicated; it is the region for which both $\dot{\tau}_{1}<0$ and $\dot{\tau}_{2}<0$. Region II is the domain of the stress plane compatible with double slip when $h>h^{\prime}$; its boundary as indicated is based on eqn (7b). Regions $I 1$ and $I 2$ correspond to single slip on systems 1 and 2 , respectively, in the case $h>h^{\prime}$; the regions corresponding to single slip when $h<h^{\prime}$ are denoted by $I 1^{\prime}$ and $I 2^{\prime}$, respectively, and exhibit the overlap noted above. For the case $h^{\prime}=0$ (the slip systems harden independently), the double slip region II becomes the region where the resolved shear stress rates of both slip systems are positive, i.e. the region between the dashed lines. As the $h^{\prime}$ value grows from zero, region $I I$ shrinks and regions $I 1$ and $I 2$ of single slip expand. When $h^{\prime}=h$, region $I I$ degenerates to a line and then only a pure tensile load can activate both systems. As $h^{\prime}$ grows larger than $h$, a region $I I$ of different character expands from the line and, according to eqns (5) and (6), the regions of single slip, now denoted by $I 1^{\prime}$ and $I 2^{\prime}$, overlap region $I I$. Hence, the crystal responds nonuniquely in this new region II. For example, if we arbitrarily assume that for a stress rate in this region II both slip systems are activated, then the plastic shear strain rate $\dot{\epsilon}_{12}^{p}$ is found to be of opposite sign to the applied shear stress rate $\dot{\sigma}_{12}$, since it is easy to show (Asaro[9])

$$
\dot{\epsilon}_{12}^{P}=\cos ^{2} 2 \phi \dot{\sigma}_{12} /\left(h-h^{\prime}\right)
$$

when both systems are active. On the other hand, one may verify that an alternate single slip solution exists such that if $\dot{\sigma}_{11}, \dot{\sigma}_{12}$ is directed into region II and $\dot{\sigma}_{12}>0(<0)$, then only system $2(1)$ is active, $\dot{\gamma}_{1}=0\left(\dot{\gamma}_{2}=0\right)$, and $\dot{\epsilon}_{12}^{P}>O(<0)$. This non-uniqueness of response to a given stress-rate direction is not resolvable within the rate-independent model although we show later that the rate-dependent model, phrased in a manner consistent with $h^{\prime}>h$, tends to select the single-slip mode. As the full analysis of Appendix 2 shows, when $\sigma_{11}$ is not negligible compared to $h$, the switch from unique to non-unique response in region II occurs when $h^{\prime}>h / K$, in terms of the rotational factor $K$ introduced there, and this may be rewritten as the condition

$$
h^{\prime}>h+2 \sigma_{11} \sin ^{2} \phi \cos 2 \phi \text {. }
$$

When $\phi=45^{\circ}$, the shear stress $\sigma_{12}$ makes no contribution to the resolved shear stress on buth systems. This is the trivial case. Since in this case the lines $\tau_{1}=$ const. and $\tau_{2}=$ const. are 
colinear and all the vertex structures shown in Fig. 2(b) will degenerate to a vertical line, the yield surface is smooth.

When $\phi>45^{\circ}$, the relative positions of the lines labelled $\tau_{2}=$ const. and $\tau_{1}=$ const. in Fig. 2(b) change. The inequality for the case that slip system 2 is active and that slip system 1 is inactive is

$$
\dot{\sigma}_{12} \leq \frac{1}{2} \tan 2 \phi \frac{h-h^{\prime}}{h+h^{\prime}} \dot{\sigma}_{11}
$$

and the inequality for the case that slip system 1 is active and that slip system 2 is inactive is

$$
\dot{\sigma}_{12} \geq-\frac{1}{2} \tan 2 \phi \frac{h-h^{\prime}}{h+h^{\prime}} \dot{\sigma}_{11}
$$

If we plot the crystal's response in stress space, we will have the reflection of Fig. 2(b) through the $\sigma_{11}$ axis. The qualitative results are the same as those for $\phi<45^{\circ}$.

Now, within the rate-dependent Schmid-like framework, we assume that for a given slipped state, the rate of plastic shearing $\dot{\gamma}_{i}$ on slip system $i$ (of group $i=1,2, \ldots, n$ ) depends on the current stress state only through the shear stress $\tau_{i}$ resolved onto system $i$. Symbolically,

$$
\dot{\gamma}_{i}=\dot{\gamma}_{i}\left(\tau_{i} \text {, current state }\right), i=1,2, \ldots, n \text {. }
$$

Prior plastic shearing of all slip systems intersecting a given point may affect the current response of a particular slip system at that point. As a specific basis for calcualation, not necessarily having a direct microphysical foundation, we consider the power-law non-linear viscous form

$$
\dot{\gamma}_{i}=\dot{a}_{i}\left(\frac{\tau_{i}}{\left.g_{i} \text { (current state }\right)}\right)^{1 / m} .
$$

Here the constants $\dot{a}_{i}$ may be regarded as reference shearing rates, such that if the crystal is to be deformed with each $\dot{\gamma}_{i}$ set equal to $\dot{a}_{i}$, then $\tau_{i}=g_{i}$ describes the requisite shear stress resolved on the $i$ th slip system. The functions $g_{i}$, by analogy with eqn (4), are then supposed to be given by

$$
\dot{g}_{i}=\sum_{j=1}^{n} h_{i j} \dot{\gamma}_{j}, i=1,2, \ldots, n
$$

The exponent $1 / m$ is consistent with a plastic strain rate sensitivity parameter defined as

$$
m=\frac{\partial \ln \tau}{\partial \ln \ddot{\gamma}}
$$

In our analysis, $m$ is taken as a small positive constant, which is the same for all slip systems.

For the double slip model $i=1,2$ and we write

$$
\begin{aligned}
& \dot{g}_{1}=h \dot{\gamma}_{1}+h^{\prime} \dot{\gamma}_{2} \\
& \dot{g}_{2}=h^{\prime} \dot{\gamma}_{1}+h \dot{\gamma}_{2} .
\end{aligned}
$$

For small shears $\gamma_{1}$ and $\gamma_{2}$ beyond a state to which the crystal was loaded by uniaxial tension up to the stress $\sigma_{11}^{0}$, with both slip systems responding at the fixed rate $\dot{a}_{1}=\dot{a}_{2}=\dot{a}$, we can write

$$
\begin{aligned}
& g_{1}=\frac{1}{2} \sigma_{11}^{0} \sin 2 \phi+h \gamma_{1}+h^{\prime} \gamma_{2} \\
& g_{2}=\frac{1}{2} \sigma_{11}^{0} \sin 2 \phi+h^{\prime} \gamma_{1}+h \gamma_{2}
\end{aligned}
$$


(these are valid for arbitrary $\gamma_{1}$ and $\gamma_{2}$ if $h$ and $h^{\prime}$ are constant). The integration constant $(1 / 2) \sigma_{11}^{0} \sin 2 \phi$ is the resolved shear stress on both slip systems at the initial state considered, which we take to be that just before the inception of loading by $\sigma_{12}$. A superscript " 0 " denotes the quantity at the instant just before the inception of $\sigma_{12}$ loading.

If the stress rates $\dot{\sigma}_{11}, \dot{\sigma}_{12}$ remain constant after the inception of shear, eqn (9) becomes

$$
\begin{aligned}
& \dot{\gamma}_{1}=\dot{a}\left(\frac{(1 / 2) \sigma_{11}^{0} \sin 2 \phi+\left((1 / 2) \dot{\sigma}_{11} \sin 2 \phi-\dot{\sigma}_{12} \cos 2 \phi\right) t}{(1 / 2) \sigma_{11}^{0} \sin 2 \phi+h \gamma_{1}+h^{\prime} \gamma_{2}}\right)^{(1 / m)} \\
& \dot{\gamma}_{2}=\dot{a}\left(\frac{(1 / 2) \sigma_{11}^{0} \sin 2 \phi+\left((1 / 2) \dot{\sigma}_{11} \sin 2 \phi+\dot{\sigma}_{12} \cos 2 \phi\right) t}{(1 / 2) \sigma_{11}^{0} \sin 2 \phi+h^{\prime} \gamma_{1}+h \gamma_{2}}\right)^{(1 / m)}
\end{aligned}
$$

where $t$ is time since inception of shear loading.

We rearrange eqns (15) and (16) to a dimensinless form as

$$
\begin{aligned}
& \frac{\mathrm{d} \Gamma_{1}}{\mathrm{~d} \theta}=\left(\frac{1+B\left(1-2\left(\mathrm{~d} \sigma_{12} / \mathrm{d} \sigma_{11}\right) \cot 2 \phi\right) \theta}{1+\Gamma_{1}+\left(h^{\prime} / h\right) \Gamma_{2}}\right)^{(1 / m)} \\
& \frac{\mathrm{d} \Gamma_{2}}{\mathrm{~d} \theta}=\left(\frac{1+B\left(1+2\left(\mathrm{~d} \sigma_{12} / \mathrm{d} \sigma_{11}\right) \cot 2 \phi\right) \theta}{1+\Gamma_{2}+\left(h^{\prime} / h\right) \Gamma_{1}}\right)^{(1 / m)}
\end{aligned}
$$

where

$$
\begin{gathered}
\Gamma_{1}=\frac{h \gamma_{1}}{(1 / 2) \sigma_{11}^{0} \sin 2 \phi}, \Gamma_{2}=\frac{h \gamma_{2}}{(1 / 2) \sigma_{11}^{0} \sin 2 \phi} \\
\theta=\frac{h \dot{a} t}{(1 / 2) \sigma_{11}^{0} \sin 2 \phi}, \quad B=\frac{(1 / 2) \dot{\sigma}_{11} \sin 2 \phi}{h \dot{a}}=\frac{\dot{\sigma}_{11}}{\dot{\sigma}_{11}^{0}}\left(1+h^{\prime} / h\right) .
\end{gathered}
$$

Equations (17) and (18) show that results depend only on $\mathrm{d} \sigma_{12} / \mathrm{d} \sigma_{11}, h^{\prime} / h$, and $B$ for a given slip angle $2 \phi$. For the numerical solutions, we arbitrarily choose $2 \phi=70^{\circ}$ to be representative of the slip angle of a face centered cubic crystal[9] (we note that the slip angle $2 \phi$ may be nearer to $120^{\circ}$ for a body centered cubic crystal[9]). We also keep the tensile stress rate constant along the deformation history (i.e. $\dot{\sigma}_{11}=\dot{\sigma}_{11}^{0}=$ constant, so that $B=1+h^{\prime} / h$ ).

Equations (17) and (18) have been integrated numerically. The results are plotted in Figs 3(a-c) and 4. In Fig. 3(a), the case of $\mathrm{d} \sigma_{12} / \mathrm{d} \sigma_{11}=1.0$ and $h^{\prime} / h=0$ is investigated. The loading direction $\mathrm{d} \sigma_{12} / \mathrm{d} \sigma_{11}$ is chosen so that both the slip systems are activated according to eqn (4). The normalized plastic shearing rates $\dot{\gamma}_{1,2} / \dot{a}$ are plotted as a function of the normalized shear stress $\sigma_{12} / \sigma_{11}^{0}$ in Fig. 3(a). The curves marked $m=0$ in all figures represent the rate-independent solution. In Fig. 3(a), we can see that, as $m$ gets smaller, the rate-dependent $\dot{\gamma}_{1,2}$ approaches the rate-independent $\dot{\gamma}_{1,2}$ faster with increasing load.

Figure 3(b) shows the numerical results for $\mathrm{d} \sigma_{12} / \mathrm{d} \sigma_{11}=2.0$ and $h^{\prime} / h=0$. The stress ratio $\mathrm{d} \sigma_{12} / \mathrm{d} \sigma_{11}$ is chosen so that only slip system 2 is activated according to the rate-independent analysis. Figure 3 (b) shows that the rate-dependent $\dot{\gamma}_{1}$ approaches zero and the rate-dependent $\dot{\gamma}_{2}$ approaches the corresponding rate-independent $\dot{\gamma}_{2}$ as load increases.

In Fig. 3(c), the numerical results are plotted for $\mathrm{d} \sigma_{12} / \mathrm{d} \sigma_{11}=0.05$ and $h^{\prime} / h=1.1$. This is the case that should result in the non-unique response of the crystal from eqn (4) since $h^{\prime}>h$. This non-uniqueness is not exhibited for the rate-dependent analysis. In Fig. $3(\mathrm{c}), \dot{\gamma}_{1.2} / \dot{a}$ is plotted as a function of $\sigma_{12} / \sigma_{11}^{0}$. The limit, as $m$ approaches zero, of the $\dot{\gamma}_{1,2}$ 's predicted from the rate-dependent analysis indicates that the crystal chooses the single slip response. Note that the two straight lines marked $m=0$ are plotted according to $\dot{\gamma}_{2}=\dot{\gamma}_{2} / \mathrm{h}$ and $\dot{\gamma}_{1}=0$.

In Fig. 4, the normalized shear stress $\sigma_{12} / \sigma_{11}^{0}$ is plotted as a function of the quantity $\epsilon_{12}^{p} h / \sigma_{11}^{0}$ for $\mathrm{d} \sigma_{12} / \mathrm{d} \sigma_{11}=1.0$ and $h^{\prime} / h=0$. Because of the viscous nature of the rate-dependent constitutive law, $\dot{\gamma}_{1.2}$ undergoes a smooth change after the inception of shear, as shown in Fig. 3(a), in contrast to the abrupt change of $\dot{\gamma}_{1,2}$ predicted by the rate-independent analysis. This continuous change in $\dot{\gamma}_{1.2}$ results in the elastic behavior (in shear) which is shown in Fig. 4. We can see that, as $m$ gets smaller, the rate-dependent results approach the much softer response (in shear) of the rate-independent analysis.

When $h^{\prime}$ is taken as zero, eqns (17) and (18) can be integrated exactly. If we further assume 


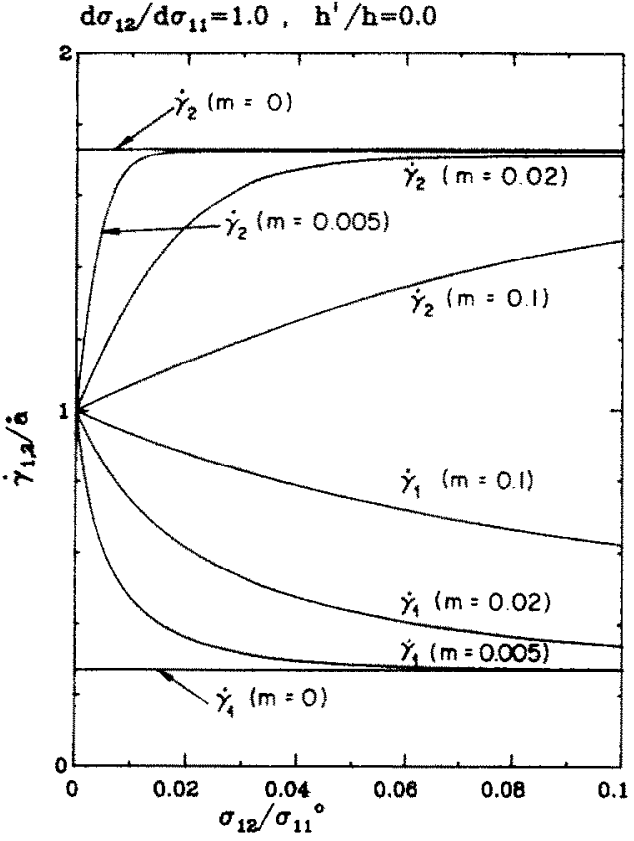

(a)

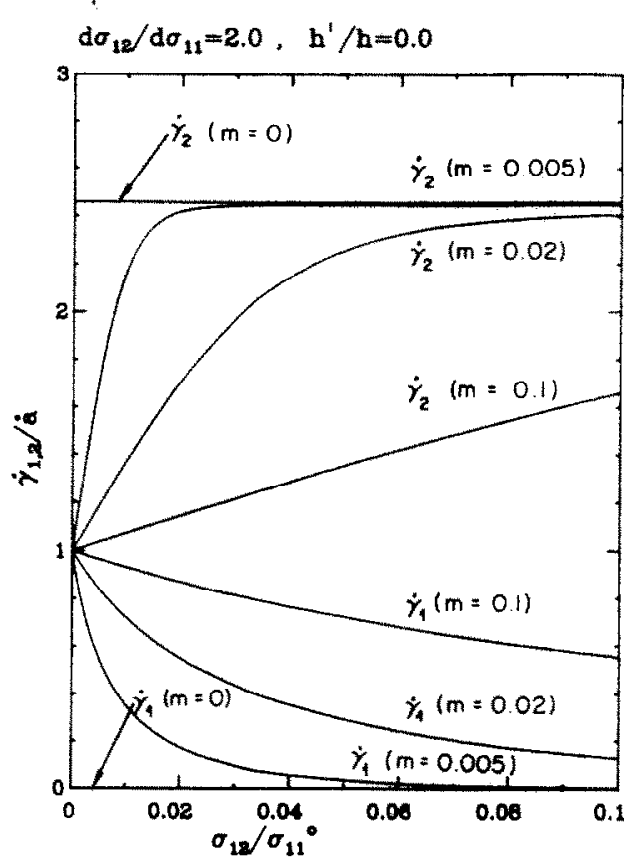

(b)

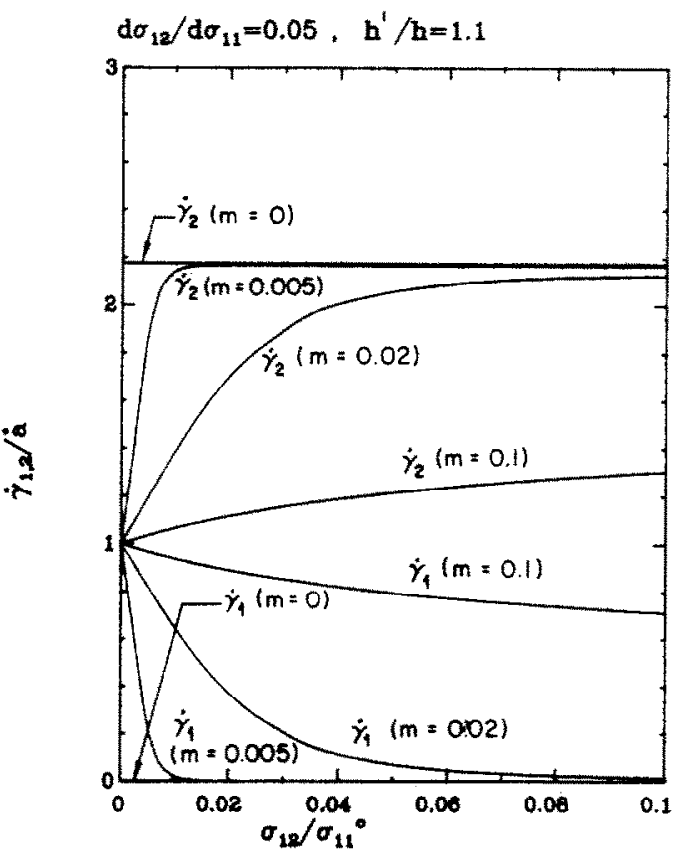

(c)

Fig. 3(a). The normalized plastic shearing rate $\dot{y}_{1.2} / a$ is ploted as a function of the normalized shear stress $\sigma_{1} / \sigma^{\prime \prime}$ for $d \sigma_{1} / d \sigma_{n}=1.0$ and $h^{i} h=0$. This stress ratio activates both slip systems according to eqn (4). (b) The normalized plastic shearing rate $\dot{y}_{1 .} / \dot{t}$ is plotied as a function of the normalized shear stress $\sigma_{1} / \sigma_{1}^{\prime}$ for $\mathrm{d} \sigma_{12} / \mathrm{d} \sigma_{11}=2.0$ and $h^{*} / h=0$. This stress ratio activates only slip system 2 according to eqn (4). (c) The normalized plastic shearing rate $\dot{\gamma}_{1.2} / \dot{a}$ is plotted as a function of the normalized shear stress $\sigma_{12} / \sigma_{11}^{0}$ for $\mathrm{d} \sigma_{1:} / \mathrm{d} \sigma_{11}=0.05$ and $h^{\prime} / h=1.1$. According to eqn (4), the crystal responds non-uniquely. However. the rate-dependent analysis clearly indicates that the crystal chooses the single-slip response at the limit $m=0$.

that $\dot{\sigma}_{11}$ equals $\dot{\sigma}_{11}^{0}$, and restrict the stress ratio $\mathrm{d} \sigma_{12} / \mathrm{d} \sigma_{11}$ so that both slip systems would be activated in the $m=0$ limit, we may then expand the exact solutions around $\theta=0$. The initial crystal response in shear can therefore be obtained as

$$
\epsilon_{12}^{\mathrm{P}}=\frac{\cos ^{2} 2 \phi \sin 2 \phi}{4 m\left(\mathrm{~d} \sigma_{12} / \mathrm{d} \sigma_{11}\right)}\left(\frac{\sigma_{12}}{h}\right)^{2}
$$




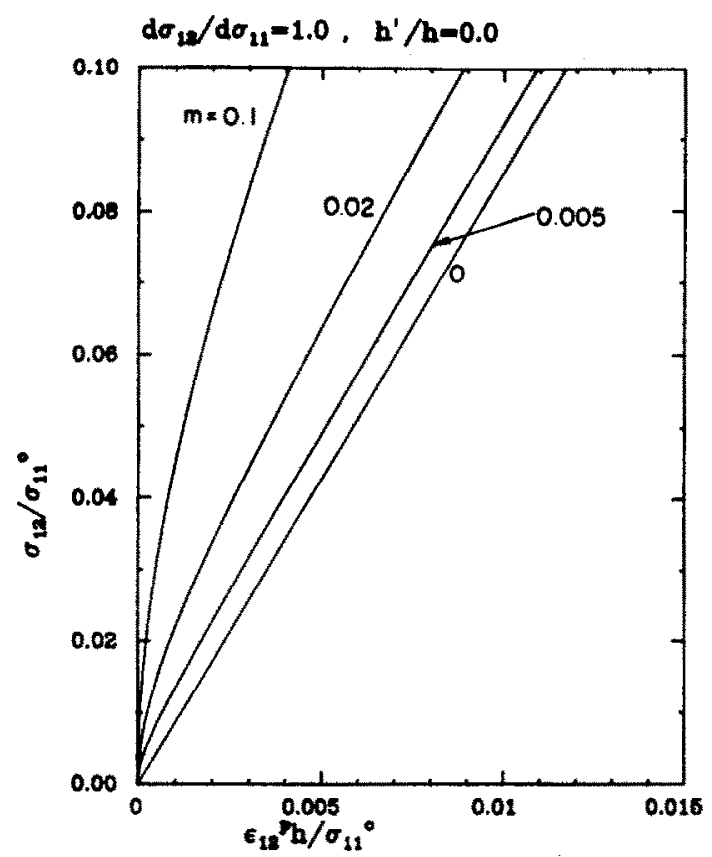

Fig. 4. The normalized shear stress $\sigma_{12} / \sigma_{11}^{\prime \prime}$ is plotted as a function of the normalized plastic shear strain $c_{1}^{\prime \prime} h / \sigma_{11}^{\prime \prime}$ for $\mathrm{d} \sigma_{1} / \mathrm{d} \sigma_{11}=1.0$ and $h^{\prime} / h=0$. The rate-dependent analysis show's the elastic response in shear at the inception of shear.

Equation (19) gives the initial curvature in the plot of $\sigma_{12}$ versus $\epsilon_{12}^{p}$ after the inception of shear. The curvature is larger for smaller $m$, but the quantity $d \epsilon_{12}^{p} / \mathrm{d} \sigma_{12}$ at $\sigma_{12}=0$ is always zero which indicates that initial shear response is elastic.

Figures $3(\mathrm{a})$ and 4 show the results for $\mathrm{d} \sigma_{12} / \mathrm{d} \sigma_{11}=1.0$ and $h^{\prime} / h=0$. This is a case for which both slip systems are activated in the rate independent limit. Figure 3(a) shows that the rate-dependent $\dot{\gamma}_{1,2}$ approaches the rate-independent $\dot{\gamma}_{1,2}$ faster for smaller $m$ as deformation proceeds. There exists a steady state $-\dot{\gamma}_{i}$ become a constant after some time. This steady-state $\dot{\gamma}_{1,2}$ is denoted as $\dot{\gamma}_{1,2}^{s s}$ which is easily obtained from eqns (15) and (16) as

$$
\begin{aligned}
\frac{\dot{\gamma}_{i}^{s s}}{\dot{a}} & =\rho_{i}^{(i / 1+m)} & & \text { for } \rho_{i}>0(i=1,2) \\
& =0 & & \text { for } \rho_{i}<0
\end{aligned}
$$

where $\rho_{i}=\dot{\tau}_{i} / \dot{\tau}_{i}^{0}$. This steady state rate is essentially independent of $m$ for $m \ll 1$. Also, there are steady states seen in Fig. 3(c) for $h^{\prime} / h \neq 0$. In Fig. 4, the elastic behavior in shear and the parabolic features of the curve before reaching the steady state are observed for the ratedependent results. We can see the qualitative characteristics, that the curvature becomes small as $m$ gets large, described in eqn (19) for the rate-dependent analysis at the inception of shear.

\section{SLIP THEORY AND VERTEX YIELD EFFECTS IN POLYCRYSTALS}

Batdorf and Budiansky[13] have proposed the so-called "slip theory", the simplest physical plasticity theory for polycrystals which considers crystalline slip as the principal mechanism for plastic deformation. This version of a slip-based theory does not consider the requirements of compatibility between adjacent grains, or, stated alternatively, it neglects the residual stresses which develop due to the different slip states of each of the plastically deformed grains. (To meet the compatibility and equilibrium conditions on the microscale, Lin and Ito [14,15] used the point force solution for an infinite elastic solid in their treatment of plastic strain gradient as an equivalent body force, whereas Kroner [16], Budiansky and Wu[17] and Hill[18] developed the self-consistent models which take account of grain interactions by employing 
Eshelby's solution for an ellipsoidal inclusion undergoing shape transformation in an infinite elastic solid[19].)

The simple Batdorf-Budiansky slip theory[13], which we adopt here for analysis of the rate-dependent case, assumes that each individual grain carries the same stress as the macroscopic stress $\boldsymbol{\sigma}$. Thus, the resolved shear stress $\tau$ on a slip system having slip plane normal $\mathbf{n}$ and slip vector $s$ is

$$
\tau=\mathbf{n} \cdot \boldsymbol{\sigma} \cdot \mathbf{s}
$$

Since it lies within the framework of the classical "small strain" approach, slip theory ignores rotation of the slip systems and shear stress rates $\dot{\tau}$ are given by $\mathbf{n} \cdot \dot{\boldsymbol{\sigma}} \cdot \mathbf{s}$. Each slip system in a polycrystal is assumed to harden independently and plastic shearing of a given system is stress state dependent only through the resolved shear stress on that system. Symbolically, for the rate-independent model,

$$
\gamma=F(\hat{\tau})
$$

where $\gamma$ represents the plastic shear strain of the slip system, $\hat{\tau}$ is the maximum value of the resolved shear stress in the loading history, and $F(\tau)$ is a monotonically increasing functin which may be set equal to zero for $\tau$ less than a stress $\tau_{L}$, at which yielding is assumed to begin.

The macroscopic plastic deformation of the polycrystal is then obtained by summing $F(\hat{\tau})$ over all the possible slip systems in orientation space. We have

$$
\boldsymbol{\epsilon}^{P}=\int_{H} \int_{S} \gamma_{\Omega \beta} \frac{1}{2}(\mathbf{n s}+\mathbf{s n}) \mathrm{d} \Omega \mathrm{d} \beta
$$

where $\epsilon^{P}$ is the macroscopic plastic strain tensor, $\gamma_{\Omega \beta}$ represents the plastic shear strain of a differential element referred to the direction $\mathbf{n}$ and $\mathbf{s}$ in orientation space, $d \Omega$ is the differential solid angle about the normal $\mathbf{n}$, and $\mathrm{d} \beta$ is the differential angle about the slip vector $\mathbf{s}$. The integration is performed over a hemisphere $H$ swept out by $\mathbf{n}$, and a semicircle $S$ swept out by $\mathbf{s}$.

The function $F(\tau)$ employed in the rate-independent model can be obtained from the stress-strain curve of a simple tension test. The approximate method of [13] is to expand $F(\tau)$ as a Taylor series in $\tau-\tau_{L}$, integrate over the orientation space for the tensile plastic strain, and determine the desired number of coefficients in the series by fitting a corresponding number of points in the stress-strain curve.

Budiansky et al. conducted an experiment in which thin-walled cylinders of 14S-T4 aluminum alloy were used [8]. Those cylinders were stressed into the plastic range in axial compression and then twisted. As the twist was applied, the compression was varied in such a manner that the ratio of the increments of shear stress to the increments of compressive stress was approximately constant for a given cylinder. Figure 5 shows a portion of the compressive stress vs plastic strain relation for the material. Some of the results of the superposed shearing are shown in Figs. 6-8. In these figures, $\sigma_{11}$ denotes the compressive stress, $\sigma_{0}$ denotes the yield stress, $\epsilon_{11}^{P}$ denotes the plastic compressive strain, $\sigma_{12}$ denotes the shear stress, $\gamma_{12}^{P}\left(=2 \epsilon_{12}^{P}\right)$ is the engineering plastic shear strain, and $\Delta \epsilon_{11}^{P}$ denotes the increase of the plastic compressive strain after the inception of shear. Comparison with the predictions of the rate-independent models of $J_{2}$ flow theory, $J_{2}$ deformation theory and slip theory were made by Budiansky et al. For the positive $\mathrm{d} \sigma_{12} / \mathrm{d} \sigma_{11}$ (Figs. 6 and 7), $J_{2}$ flow theory correctly predicts the initial elastic response in shear, but it underestimates the plastic shear strain; slip theory predicts a plastic shear strain very close to that predicted by $J_{2}$ deformation theory, but both theories overestimate the plastic shear strain; the predictions of all three theories are in good agreement with the experiments for the compressive plastic strain $\epsilon_{11}^{P}$. For the negative $\mathrm{d} \sigma_{12} / \mathrm{d} \sigma_{11}$ (Fig. 8), plastic deformation occurs well before the elastic-plastic boundary, $\tau_{\text {oct }}=$ constant $\left(\tau_{\mathrm{cct}}=(2 / 3)\left(\sigma_{11}^{2}+3 \sigma_{12}^{2}\right)^{1 / 2}\right)$, of the simple flow theory is reached. Slip theory gives excellent agreement with the experimental shear strain, but underestimates the compressive plastic strain by a small amount of "creep" [8]. All rate-independent plasticity theories that are based on crystalline slip as the mechanism for 


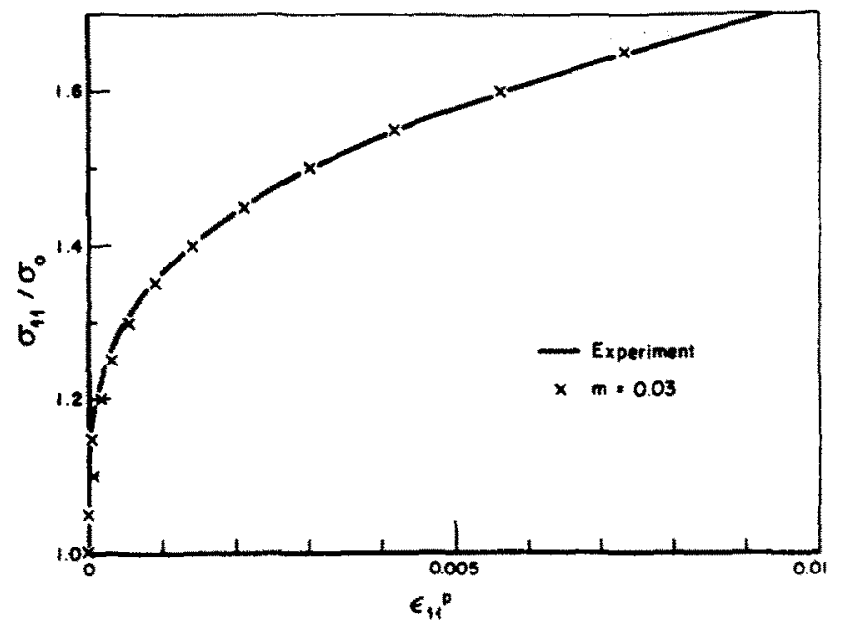

Fig 5 . The experimental results of the compression test by Budiansky $e t$ al [8] is shown. The numerical results which are calculated from the assumed $g(\gamma)$ for the rate sensitivity $m=0.03$ are also shown.

plastic deformation lead to the prediction of the vertex formation on the current yield surface. Furthermore, for the compression-shear loading path, these physical plasticity theories predict less stiffness in shear under continuous loading; the stiffness in shear approaches the elastic shear modulus as the loading direction moves from the axial compressive load to the direction in which no slip systems of the polycrystalline aggregate are activated. However, the experimental results showed the elastic stiffness at the onset of shear for all values of $d \sigma_{12} / d \sigma_{11}$. This elastic behavior in shear plainly contradicts the predictions of all slip-based plasticity theories.

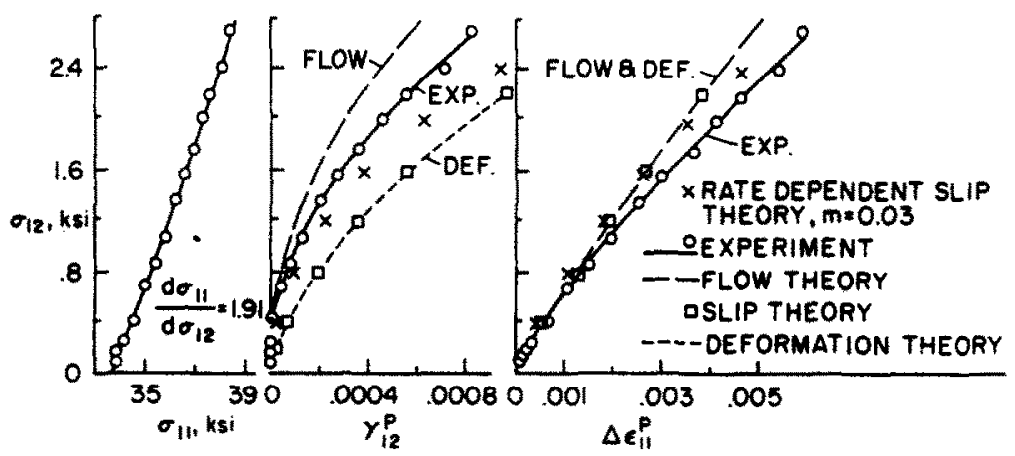

Fig. 6.

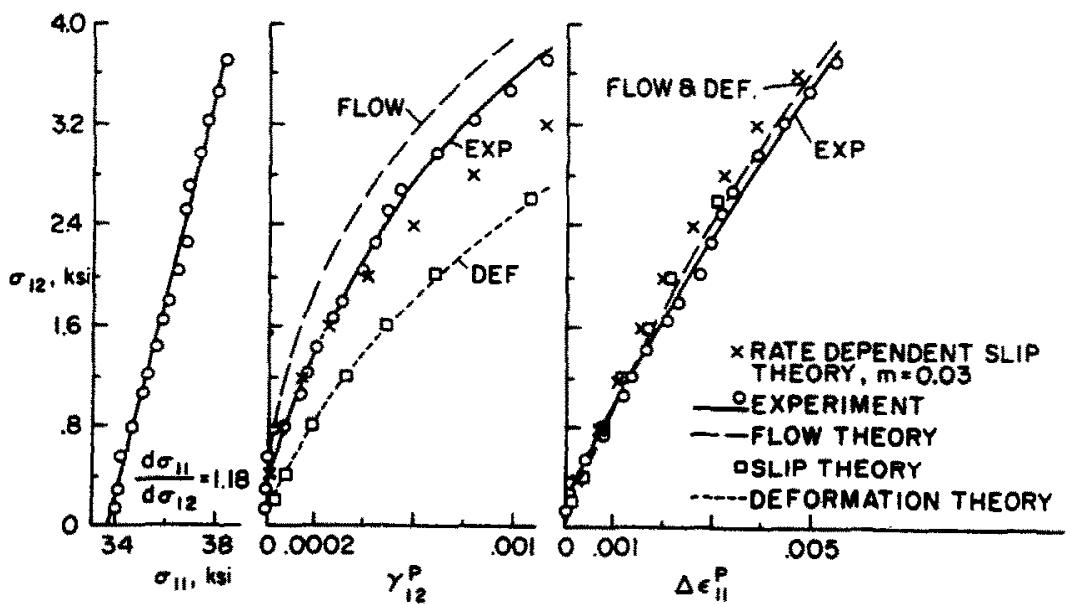

Fig. 7. 

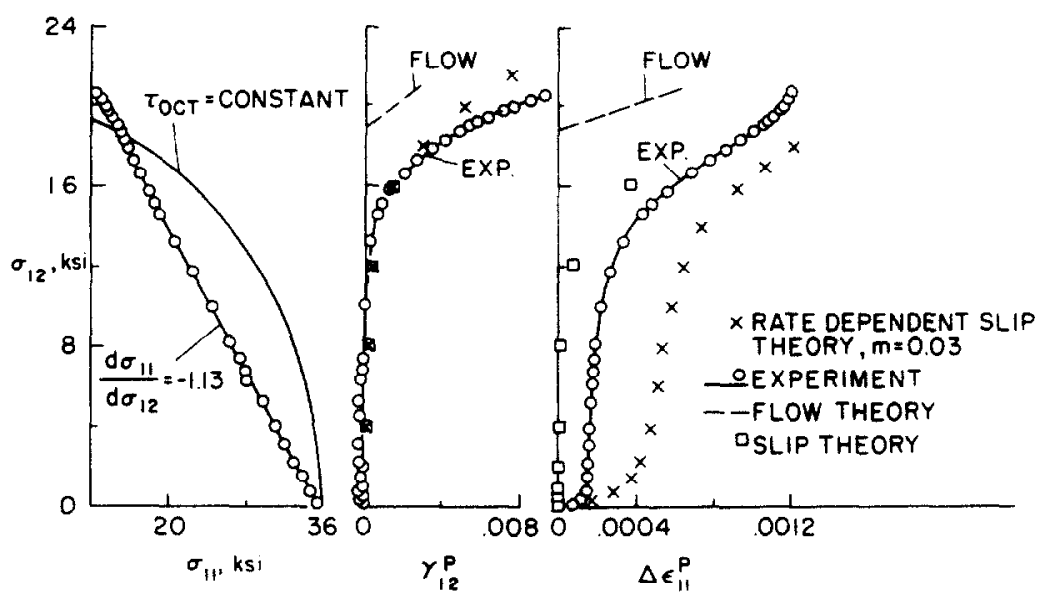

Fig. 8.

Figs. 6-8. The experimental results of the combined compression and torsion test by Budiansky et al. [8] are shown. The stress ratios $\mathrm{d} \sigma_{11} / \mathrm{d} \sigma_{12}$ are $1.91,1.18$, and -1.13 for Figs, 6.7 and 8 , respectively. The predictions by various theories are compared. The rate sensitivity $m$ of the rate-dependent slip theory is 0.03 for all cases. The elastic response (in shear) predicted by the rate-dependent slip theory is noted. Also. the "creep" compression strain is predicted by the rate-dependent slip theory.

Now, if we assume that the slip processes are rate-dependent and that the slip rates vary continuously with the resolved shear stresses, we can predict the elastic response in shear for the above-mentioned compression-torsion tests by the argument of Rice[6]. Within the context of slip theory, the non-linear viscous form is again used to describe the slip process of a slip system, viz,

$$
\dot{\gamma}=\dot{a}\left(\frac{\tau}{g(\gamma)}\right)^{1 / m}
$$

where $\dot{\gamma}$ is the plastic shearing rate, $\dot{a}$ is the reference plastic shearing rate, $m$ represents the plastic strain rate sensitivity, and $g(\gamma)$ is the function of the current state which is taken as the plastic shear strain $\gamma$ of the slip system because of the assumption of independent hardening (Note that $\tau=g(\gamma)$ when $\dot{\gamma}=\dot{a}$.) The plastic shear strain $\gamma$ of each slip system is thus obtained by integrating eqn (23) incrementally. The macroscopic plastic strain is then obtained by integrating the plastic shear strain of all the slip systems in the orientation space as in eqn (22).

For a given rate sensitivity $m$, the function $g(\gamma)$ is found by fitting the numerical results of the pure compression curve, Fig. 5, to the experimental data. Several assumptions are made in the numerical calculations. Here, the compressive stress rate $\dot{\sigma}_{11}$ is assumed to be a constant to simplify calculation of the material response in pure compression, whereas the compressive strain rate was kept constant in the experiments. A monotonically increasing function $g(\gamma)$ with $g(0)=\tau_{L}$ is guessed; the load is applied, and when the value of the shear stress $\tau$ resolved on the slip system at an integration point is first detected to be larger than $\tau_{L}$, which is equal to half the value of the yield stress in the pure compression test, the plastic shear strain $\gamma$ of this slip system is assumed to equal $g^{-1}(\tau)$ (the inverse function of $g(\gamma)$ ) which is a very small value near zero. The reference shearing rate $\dot{a}$ of this slip system is then assumed to be $g^{-1}(\tau) \tau$ (where $g^{-1}(\tau)$ means the derivative of $g^{-1}(\tau)$ with respect to $\tau$ ) such that the constitutive law, eqn (23), can be satisfied at this moment. The plastic shear strain $\gamma$ of the slip system is then integrated incrementally according to eqn (23) as deformation proceeds. For those integration points at which the resolved shear stress $\tau$ is never larger than $\tau_{L}$, the plastic shear strain $\gamma$ is simply taken as zero. The above assumptions are justified by the fact that plastic deformation is very small below the "yield stress" (from the viewpoint of rate-independent plasticity) in an ordinary compression test. Therefore, we assume that the amount of slip can be neglected until $\tau$ reaches $\tau_{L}$. For a fixed value of $m$, a $g(\gamma)$ can be found such that the compression plastic response matches the experimental compression test. In Fig. 5, the experimental plastic 
compressive strain is plotted as a solid line, and the well-matched numerical results which are claculated by the guess $g(\gamma)$ are also plotted for $m=0.03$.

The numerical results of the combined compression-torsion test based on the guess $g(\gamma)$ were obtained for $m=0.005,0.01,0.03$ and 0.05 . In those calculations, we assumed that $\dot{\sigma}_{11}=\dot{\sigma}_{11}^{0}=$ constant for a positive value of $\mathrm{d} \sigma_{11} / \mathrm{d} \sigma_{12}$, and $\dot{\sigma}_{11}=-\dot{\sigma}_{11}^{0}=$ constant for the negative value of $\mathrm{d} \sigma_{11} / \mathrm{d} \sigma_{12}$. The numerical results for $m=0.03$ are shown with the cross symbols in Figs. 6-8.

In these figures, the rate-dependent results do show the elastic response in shear at the inception of shear. However, as the shear stress becomes large, the present numerical results overestimate the plastic shear strain for the positive values of $\mathrm{d} \sigma_{11} / \mathrm{d} \sigma_{12}$, and they underestimate this strain for the negative value of $\mathrm{d} \sigma_{11} / \mathrm{d} \sigma_{12}$. The compressive plastic strain of the numerical results matches the experimental results well for the positive values of $\mathrm{d} \sigma_{11} / \mathrm{d} \sigma_{12}$; but, for the negative value of $\mathrm{d} \sigma_{11} / \mathrm{d} \sigma_{12}$, the numerical results overestimate the compressive plastic strain by some amount of creep. However, the qualitative trend is consistent with the experimental data.

Although the numerical results for $m$ other than 0.03 are not shown here, these results are summarized in the following. The rate-dependent numerical results approach to those of the slip theory of Batdorf and Budiansky when $m$ becomes small. The compressive plastic strain of the numerical results for all values of $m$ matches the experimental data well for the positive $\mathrm{d} \sigma_{11} / \mathrm{d} \sigma_{12}$ This might be due to the fact that the input parameters to the rate-dependent slip model are obtained so that the stress-strain relation in pure compression of the rate-dependent slip model matches that of the experimental results. The compressive plastic strain of the numerical results for $m=0.01$ matches the experimental data very closely for the negative $\mathrm{d} \sigma_{11} / \mathrm{d} \sigma_{12}$. As $m$ gets larger, the predicted amount of creep in compression becomes larger. However, the qualitative trend is still consistent with the experimental data as shown in Fig. 8 for $m=0.03$.

The initially elastic response in shear is shown for all values of $m$. For $m$ as small as 0.005 , the shear response of the numerical results is very close to that of the slip theory of Batdorf and Budiansky. This is due to the fact that the rate-dependent numerical results approach to those of the rate-independent slip theory when $m$ becomes small. Consequently, the curvature at $\gamma_{12}^{p}=0$ becomes large as $m$ gets small. This is consistent with the prediction of twodimensional double slip model of a single crystal as expressed in eqn (19) and as shown in Fig. 4. For the negative $\mathrm{d} \sigma_{11} / \mathrm{d} \sigma_{12}$ the plastic shear strain of the numerical results for all values of $m$ matches the experimental data except for some underestimation of $\gamma_{12}^{p}$ in the range where $\sigma_{12}$ is larger than $16 \mathrm{ksi}$.

Apparently the value $m=0.03$, chosen here as providing a reasonable fit to the data for 14S-T4 aluminum alloy, is rather high. The rate sensitivity $m$ of aluminum alloys is typically in the range of $-0.005-0.005$ as reported in [21]. Nevertheless, our numerical results for $m=0.03$ shown in Figs. 6-8 demonstrate the qualitative features due to rate sensitivity of plastic flow for a polycrystalline material under a nonproportional loading condition. Perhaps a more elegant finite deformation model, which should take account of residual stress, rotation of the slip systems, and latent hardening of the slip systems, is needed to explore thoroughly the plastic behavior of the polycrystal under more complex loading conditions.

\section{DISCUSSION}

When crystalline slip is considered as the principal mechanism for plastic deformation, formation of a vertex on the current yield surface is predicted by a rate-independent analysis. We have shown that small material rate sensitivity, essentially ignorable in proportional loading experiments, can furnish a reasonable explanation of some experimental results, such as those of the combined compression-torsion tests, which have not demonstrated such a vertex structure.

In the analysis of a single crystal deforming in a double slip pattern, assuming a rate-dependent slip process and allowing the material rate sensitivity to approach zero was shown to help to select a deformation mode, whereas this mode could not be determined uniquely through the rate-independent approach.

In the polycrystal model based on the slip concepts of Batdorf and Budiansky, the function $g(\gamma)$ and the rate sensitivity $m$ in the viscous slip law (eqn 23) is obtained from a trial-and-error 
method by fitting the compressive plastic response of a compression test. The choices of $g(\gamma)$ and especially $m$ are rather arbitrary; however, the effort we make here is to show that the plastic strain rate changes smoothly for the rate-dependent approach even though the stress rate has a jump, and that the elastic behavior in shear of the combined compression-torsion tests can be predicted with this simple rate-dependent model based on the slip theory of Batdorf and Budiansky.

Our study supports the idea of vertex formation on the current yield surface, in the sense that this does result in the rate-insensitive limit of a rate-dependent analysis. While deformation theory, some phenomenological corner theories, and the physical plasticity theories, which are all suggestive of the existence of a vertex (or corner) formed on the current yield surface, are extensively used in bifurcation or buckling analyses in the rate-independent sense, the retardation of instabilities due to material rate sensitivity should also be noted.

Acknowledgement-This work was done at Brown University under support of the National Science Foundation Materials Research Laboratory.

\section{REFERENCES}

1. R. Hill, The essential structure of constitutive laws for metal composites and polycrystals. J. Mech. Phys. Solids 15. $79-95$ (1967).

2. B. Paul, Macroscopic criteria for plastic flow and brittle fracture. In Fracture: An Advanced Treatise (Edited by $\mathrm{H}$. Liebowitz), Vol. 2, pp. 313-496. Academic Press, New York (1968).

3. S. S. Hecker, Constitutive Equation in Viscoplasticity: Computational and Engineering Aspects (Edited by J. A. Stricklin and R. O. Saczalski), AMD-Vol. 20, p. 1. ASME, New York (1976).

4. W. G. Johnston and J. J. Gilman, Dislocation velocities, dislocation density, and plastic flow in LiF crystals. J. Appl. Phys. 30, 129 (1959).

5. J. J. Gilman, Progress in the microdynamical theory of plasticity. Proc. 5th U.S. Nat. Congress of Appl. Mech., ASME. p. 385 (1966).

6. J. R. Rice, On the structure of stress-strain relations for time-dependent plastic deformation in metals. Trans ASME, J. Appl. Mech. 37. 728 (1970).

7. J. R. Rice. Inelastic constitutive relations for solids: an internal-variable theory and its application to metal plasticity. J. Mech. Phys. Solids 19, 433-455 (1971).

8. B. Budiansky, N. Dow, R. Peters and R. P. Shepherd, Experimental studies of polyaxial stress-strain laws of plasticity. Proc. 1st U.S. Nat. Cong. Appl. Mech. pp. 503-512. ASME. New York (1951).

9. R. J. Asaro, Geometrical effects in the inhomogeneous deformation of ductile single crystals. Acta Metallurgica 27. 445-453 (1979).

10. R. J. Price and A. Kelly, Deformation of age-hardened aluminum alloy crystals-II. Fracture. Acta Metallurgica 12. 979-992 (1964).

11. C. N. Reid. Deformation Geometry for Material Scientists. Pergamon Press, Oxford (1973).

12. R. Hill, Generalized constitutive relations for incremental deformation of metal crystals by multislip. J. Mech. Phys. Solids 14, 95-102 (1966).

13. S. B. Batdorf and B. Budiansky. A mathematical theory of plasticity based on the concept of slip. NACA TN 1871 (1949).

14. T. H. Lin and M. Ito, Theoretical plastic distortion of a polycrystalline aggregate under combined and reversed stresses. J. Mech. Phys. Solids 13, 103-115 (1965).

15. T. H. Lin and M. Ito, Theoretical plastic stress-strain relationship of a polycrystal and the comparisons with the von Mises and the Tresca plasticity theories. Int. J. Engng Sci. 4, 543-561 (1966).

16. E. Kroner, On the plastic deformation of polycrystals. Acta Metallurgica 9, 155-161 (1961),

17. B. Budiansky and T. T. Wu. Theoretical prediction of plastic strains of polycrystals. Proc. 4th U.S. Nat. Cong. Appl. Mech., pp. 1175-1185. ASME, Neu York (1962).

18. R. Hill, Continuum micro-mechanics of elastoplastic polycrystals. J. Mech. Phys. Solids 13, 89-101 (1965).

19. J. D. Eshelby, The determination of the elastic field of an ellipsoidal inclusion. and related problems. Proc. Roy. Soc Lond. A241, 376-396 (1957)

20. J. W. Hutchinson, Elastic-plastic behavior of polycrystaline metals and composites. Proc. Roy. Soc. Lond. A319. 247-272 (1970)

21. A. K. Ghosh, The influence of strain hardening and strain-rate sensitivity on sheet metal forming. Trans. ASME, J. Engng Mat. Tech. 264-274 (1977).

\section{APPENDIX 1}

The reason for the predicted yield surface vertex structure. when rate-independent crystalline slip is assumed as a micromechanism, is most readily seen when the problem is addressed within classical "small strain" concepts such that alternations of the local geometry of the polycrystalline array are ignored, local elastic response is tinear, and local elastic moduli within each erystalline element are assumed to be unaffected by slip. Then (e.g. Hill[1]. Rice[6]) the local resolved shear stress $\tau$ on any particular slip system, at any point of some crystalline element (grain) of the array has the form

$$
\tau=c+n_{i j} \sigma_{i j}
$$

where $\sigma_{i j}$ is the macroscopic stress state. Here $n_{i j}$ and $c$ are independent of $\sigma_{i j}, n_{i j}$ depends only on the local point and system considered but not on the distribution of plastic shear within the array, whereas $c$ depends additionally on the 
distribution of shears (and represents the "residual" shear stress that would remain if $\sigma_{i j}$ were reduced to zero under conditions for which the grains were constrained against further plastic shearing). According to the Schmid criterion, yield occurs at the point and on the system considered when $\tau$ attains a critical value $\tau_{c}$ which may, in general, be a functional of the prior plastic shears experienced. Hence the local yield criterion $\tau=\tau_{i}$, or

$$
c+n_{i j} \sigma_{i j}=\tau_{\text {. }}
$$

maps as a plane in stress space with orientation $n_{i j}$ : as plastic distrotion of the polycrystalline array takes place, this plane may translate since $\tau_{\mathrm{s}}$ and $c$ depend on the ongoing plastic shears, but it does not change orientation ( $n_{i j}$ is constant). Evidently, the macroscopic yield criterion is the inner envelope of local planar yield surfaces for all possible slip systems and all points of the polycrystalline array. Further, during a general program of plastic distortion of the polycrystal. the (translating) planar yield surfaces for each plastically active slip system and point of the array must pass through the current stess point (Fig. 1). At conditions well into the plastic range, this collection of planes encompasses a wide range of orientations $n_{\mathrm{ij}}$, and hence a pronounced yield surface vertex is developed. Specific calculations of subsequent yield surfaces for polycrystals. necessarily exhibiting the vertex structure, are given by Lin and Ito[14, 15] and Hutchinson[20].

\section{APPENDIX 2}

When a rigid plastic single crystal deforms in a single slip pattern. the angle between the slip direction and the tensile axis, $\phi$, decreases due to the constraints of the specimen grips. As in Asaro[9], we have the relationship

$$
\dot{\phi}=-\tan \phi \dot{\epsilon}_{11}^{P}=-\sin ^{2} \phi \dot{\gamma}
$$

At the inception of shear under double slip conditions, the resolved shear stress rates of both slip systems are

$$
\begin{aligned}
& \tau_{1}=\frac{1}{2} \dot{\sigma}_{11} \sin 2 \phi_{1}-\dot{\sigma}_{12} \cos 2 \phi_{1}+\sigma_{11} \dot{\phi}_{1} \cos 2 \phi_{1} \\
& \tau_{2}=\frac{1}{2} \dot{\sigma}_{11} \sin 2 \phi_{2}+\dot{\sigma}_{12} \cos 2 \phi_{2}+\sigma_{11} \dot{\phi}_{2} \cos 2 \phi_{2}
\end{aligned}
$$

where $\phi_{1}$ denotes the angle between the slip vector $s_{1}$ and the tensile axis $x_{1}$, and $\phi_{2}$ denotes the angle between the slip vector $s_{2}$ and the tensile axis $x_{1}$. At the inception of shear, $\phi_{1}$ is equal to $\phi_{2}$ and is denoted as $\phi$.

When $\phi<45^{\circ}$, we consider the case that slip system 1 is active and slip system 2 is not active. Thus,

$$
\dot{\phi}_{1}=-\sin ^{2} \phi \dot{\gamma}_{1}=-\dot{\phi}_{2}
$$

From eqns (A2)-(A4), and (4). we obtain the inequality,

$$
\dot{\sigma}_{12} \leq-\frac{1}{2} \frac{h-h^{\prime} K}{h+h^{\prime} K} \tan 2 \phi \dot{\sigma}_{11}
$$

where

$$
K=\frac{1-\left(\sigma_{11} / h^{\prime}\right) \sin ^{2} \phi \cos 2 \phi}{1+\left(\sigma_{11} / h\right) \sin ^{2} \phi \cos 2 \phi}
$$

Similarly, we have another inequality for the case that slip system 2 is active and slip system 1 is inactive, viz.

$$
\dot{\sigma}_{12} \geq \frac{1}{2} \frac{h-h^{\prime} K}{h+h^{\prime} K} \tan 2 \phi \dot{\sigma}_{11}
$$

Equation (A5) and (A6) are similar to eqns (5) and (6) except that a factor $K$ appears along with $h^{\prime}$. Since $h$ and $h^{\prime}$ are larger than or comparable to $\sigma_{11}$, the value of $K$ is less than unity. When $h$ and $h^{\prime}$ are much larger than $\sigma_{11}$, the value of $K$ approximately equals I: thus, the rotation of the slip systems can be neglected. When $h$ and $h^{\prime}$ are comparable to $\sigma_{11}$. the value of $K$ is no longer near to 1 , and the rotation of the slip systems should be considered. Without consideration of the rotation of the slip systems, the conditions that a nonunique region in stress space exists is $\boldsymbol{h}^{\prime}>\boldsymbol{h}$. With consideration of the rotation of the slip systems, the condition mentioned above becomes $h^{\prime} K>h$. Since $K$ can be significantly less than 1 when stress $\sigma_{11}$ is of the same order as $h$ (and $\cos 2 \phi>0$ ), the crystal seems to be much more stable with respect to loss of uniqueness under those conditions.

When $\phi>45^{\circ}$, the inequality for the case that slip system $I$ is active and that slip system 2 is inactive is

$$
\dot{\sigma}_{12} \geq-\frac{l}{2} \frac{h-h^{\prime} K}{h+h^{\prime} K} \tan 2 \phi \dot{\sigma}_{11}
$$

and the inequality for the case that slip system 2 is active and that slip system 1 is inactive is

$$
\dot{\sigma}_{12} \leq \frac{1}{2} \frac{h-h^{\prime} K}{h+h^{\prime} K} \tan 2 \phi \dot{\sigma}_{11}
$$

When $h$ and $h^{\prime}$ are much larger than $\sigma_{11}$, the value of $K$ approximately equals l: the rotation of the slip system can be neglected. When $h$ and $h^{\prime}$ are comparable to $\sigma_{11}, K$ can be larger than $l(\cos 2 \phi<0)$; the crystal is more unstable as far as loss of uniqueness (when $h^{\prime} K>h$ ) is concerned. 